\title{
MORFOGÉNESIS, MORFODINÁMICA Y CARACTERI- ZACIÓN TÉRMICA DE MONTÍCULOS DE HIELO EN LOS PIRINEOS (MACIZOS DE MALADETA Y POSETS)
}

\author{
María GONZÁLEZ GARCÍA (mariagon@uma.es) \\ GIR PANGEA, Universidad de Valladolid \\ Enrique SERRANO CAÑADAS (serranoe@,fyl.uva.es) \\ Departamento de Geografía, Universidad de Valladolid, Valladolid \\ Juan José GONZÁLEZ TRUEBA (ijgtrueba@,hotmail.com) \\ Departamento de Estudios Culturales, CIESE Comillas-Universidad de Can- \\ tabria
}

Recibido: $23 / 12 / 2015$

Aceptado: 10/06/2016

RESUMEN: Los montículos de hielo son formas periglaciares localizadas en los macizos de la Maladeta y Posets (Pirineos Centrales) entre 2670 y 2900 metros de altitud. Se ha realizado el inventariado y análisis geomorfológico de detalle complementado con registros térmicos continuos del suelo y medidas de la temperatura basal del manto nival (BTS) durante el período 2009-2011. Todo ello permite establecer la dinámica actual de estas formas periglaciares. El seguimiento térmico muestra el comportamiento térmico anual y permite interpretar su morfogénesis. Los factores topoclimáticos, el permafrost y el hielo estacional determinan su génesis, localización y durabilidad, y se han localizado montículos de hielo en diferentes altitudes, emplazamientos geomorfológicos y condiciones térmicas. En este trabajo se establecen distintos tipos en ambientes morfogenéticos diferenciados: «montículos de hielo en áreas con permafrost», donde la emisión de frío es directa desde el subsuelo, en este caso el ambiente con permafrost puede proceder de la presencia de un helero enterrado, capaz de emitir una onda de frío $\mathrm{y}$ «montículos de hielo en áreas con hielo estacional», donde la emisión de frío es indirecta, con frío procedente de los factores ambientales y topoclimáticos. La localización en un ambiente marginal de alta montaña templada como son los Pirineos, les convierte en un eficaz geoindicador morfoclimático en relación directa con las variaciones térmicas interanuales.

PALABRAS CLAVE: montículo de hielo, régimen térmico, periglaciar, permafrost, Pirineos.

GENESIS, DYNAMIC AND THERMAL CHARACTERIZATION OF FROST MOUNDS IN THE PYRENEES (MALADETA AND POSETS MASSIFS)

ABSTRACT: The frost mound are periglacial landforms located between 2670 and $2900 \mathrm{~m}$ a.s.l. An inventory of landforms and the geomorphological analysis have been made, completed by BTS measurements and continuous datalogger of soil temperature during 2009-2011 period. The genesis and present day dynamic of frost mounds and thermal behaviour of ground have been analysed. The topoclimatic factors, permafrost environment and the seasonal frozen ground determine the genesis, place and conservation of frost mounds in the Pyrenees. We have 
differentiate between «Frost mounds on permafrost environments and buried ice», where the existence of ice masses and frozen bodies as rock glaciers determine his genesis; or «frost mounds on seasonal frozen ground areas», where the topoclimate factor are essential. The location on a marginal temperate high mountain environment implies that the landforms linked to the ground ice, as frost mounds, are efficient morphoclimate geoindicators directly related to interannual thermal variations.

KEY WORDS: Frost mounds, thermal regime, periglacial, mountain permafrost, Pyrenees.

\section{INTRODUCCIÓN}

Los montículos de hielo son formas muy poco comunes en la alta montaña pirenaica y hasta ahora han sido poco estudiadas (SERRANO et al., 2006; FEUILLET, 2010; GONZÁLEZ GARCíA, 2013). Son geoformas características de ambientes con permafrost o con suelos helados estacionales y presentan diferentes tipologías que varían en función de las características climáticas, teniendo en cuenta que las fuentes de frío pueden ser muy diversas en ambientes marginales de alta montaña templada. La formación, estructura y duración de los montículos de hielo ha sido ampliamente discutida y analizada en detalle por MACKAY (1973, 1980, 1998), WASHBURN (1973) y FRENCH (2007), entre otros autores, y en algunos casos, los pequeños montículos de hielo se han definido como pingos en miniatura (ROUJANSKI, 2008).

Las particulares condiciones térmicas atmosféricas y edáficas que caracterizan la cadena pirenaica, al igual que otros ámbitos marginales, permiten definir estas geoformas como montículos de hielo estacionales (seasonal frost mound) (EVERDingen, 1978, 2005; Pollard, 1988, 1991; FRENCH, 2007). Estos son similares a las ampollas heladas (frost blisters) estudiadas por FRENCH (2007) $y$ traducidos en el Glosario de Permafrost (EVERDINGEN, 2005) como «montículo de hielo».

Los montículos de hielo estacionales de la alta montaña templada son abultamientos métricos, compuestos por un núcleo de sedimentos helados, generado por la concentración de hielo de segregación e inyección derivado de la congelación progresiva del agua del subsuelo (FRENCH, 2007). La congelación de los sucesivos flujos de agua del subsuelo se produce por el descenso térmico atmosférico (temperatura del suelo a $0^{\circ} \mathrm{C}$ o próxima a ella durante 67 meses) o por la emisión de frío desde el subsuelo por restos de heleros enterrados, permasfrost o cuerpos helados como los glaciares rocosos. Normalmente se ubican en zonas con condiciones topoclimáticas favorables (umbrías, altitud, venteado, etc), o en ambientes con permafrost. Los montículos de hielo son formas pequeñas, que no destacan sobre el relieve, pero desde el punto de vista ambiental su presencia supone un importante geoindicador de las condiciones ambientales. Su génesis y desarrollo es la respuesta geomorfológica a las variaciones climáticas acontecidas en la montaña pirenaica, en estrecha 
relación con la deglaciación de la alta montaña, el progresivo ascenso de los pisos morfoclimáticos fríos y la evolución del medio glaciar a paraglaciar, donde proliferan las formas periglaciares.

Figura 1. Localización de las zonas de estudio.

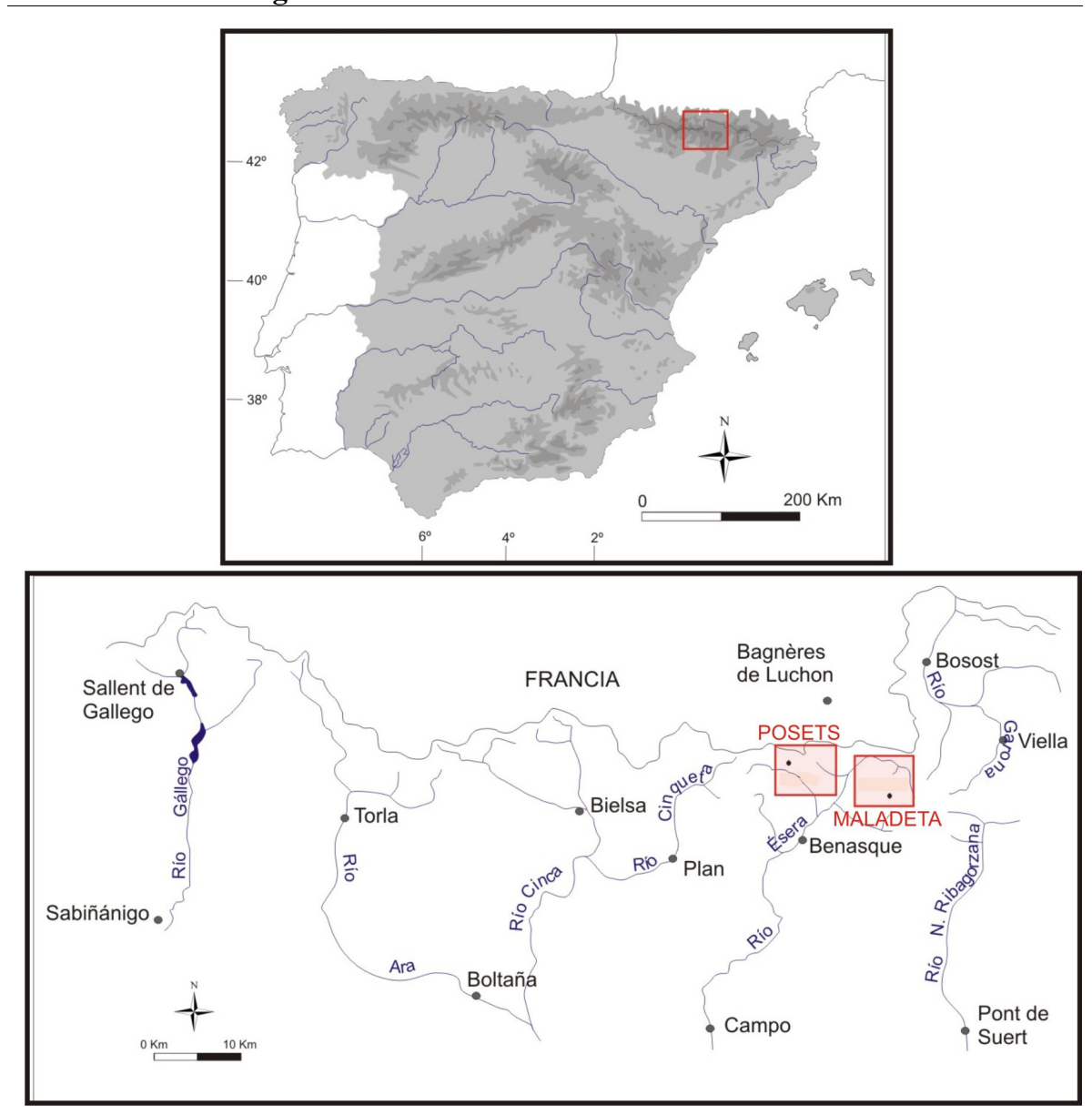

FUENTE: Los autores.

En la alta montaña pirenaica y especialmente en las zonas estudiadas se han podido distinguir diversas morfologías generadas por la crioturbación, los montículos por segregación del hielo (frost mound), suelos ordenados y microfiguraciones. En el presente trabajo analiza unas de las formas características del medio periglaciar asociada a la crioturbación. El objetivo es el estudio 
de los montículos de hielo, su localización, estructura y relación con el permafrost de montaña o los suelos helados estacionales, en dos ambientes representativos de la alta montaña pirenaica.

\section{LA ZONA DE ESTUDIO}

Los montículos de hielo estudiados se localizan en los macizos de la Maladeta y Posets (Pirineo aragonés), en la vertiente meridional del Pirineo central, y separados por el valle del río Ésera (FIGURA 1). Constituyen las máximas altitudes de la Cadena, $3.404 \mathrm{~m}$ del Pico Aneto y $3.368 \mathrm{~m}$ del Pico Posets. Bajo sus cumbres se conservan en la actualidad algunos de los glaciares más extensos de los Pirineos (glaciar del Aneto y de la Maladeta y glaciar de la Paúl) y heleros significativos (helero del Posets y helero de Alba). La deglaciación de la alta montaña pirenaica ha permitido la expansión de un medio controlado por los procesos asociados al frío y la nieve, con presencia de permafrost desde 2.760-2.780 m en Maladeta y 2.800-2.850 m en Posets, y el dominio crionival o piso periglaciar tipo desarrollado a partir de 2.600-2.700 m (SERRANO et al. 2000, 2001, 2009; GONZÁLEZ GARCÍA, 2013). La configuración morfoestructural, la masividad de ambos sectores y la elevada altitud determinan una condición favorable para la conservación de los cuerpos helados y el desarrollo de procesos y formas periglaciares.

El Macizo de la Maladeta $\left(42^{\circ} 38^{\prime} 51^{\prime \prime} \mathrm{N}-0^{\circ} 38^{\prime} 30^{\prime \prime} \mathrm{E}\right)$ forma parte de la alta cuenca del río Ésera (en el valle de Benasque), lo arman una sucesión de picos, alineados en dirección NW-SE, desde el Pico del Alba (3.118 m) al oeste hasta el pico Russell (3.207 m) al este. Se sitúa en la zona noroccidental del batolito de la Maladeta, compuesto por granodioritas, litología que le otorga un carácter masivo y su particular morfología, con una destacada disimetría morfológica (MARTÍNEZ DE PISÓN, 1986, 1989, 1990; GARCÍA-RUIZ et al. 1992). La zona de estudio se centra en el circo de Alba, que al igual que todo el conjunto montañoso, estuvo ocupado por un glaciar durante la Pequeña Edad del Hielo (en adelante PEH), hoy reducido a un helero de pequeñas dimensiones (GONZÁLEZ TRUEBA et al., 2008; GONZÁLEZ GARCÍA, 2013). Los montículos de hielo se localizan sobre los derrubios que recubren el helero de Alba y en la zona interior de la morrena frontal del glaciar de Alba, todos ellos próximos a formas que indican la existencia de permafrost como el glaciar rocoso de la Maladeta Occidental y el protalus lobe de la Maladeta (SERRANO et al., 1998, 1999, 2009, 2010b; GONZÁLEZ GARCÍA et al., 2011, 2016).

El Macizo del Posets $\left(42^{\circ} 39^{\prime} 29^{\prime \prime} \mathrm{N}-0^{\circ} 26^{\prime} 15^{\prime \prime} \mathrm{E}\right)$ se localiza entre el valle de Benasque y el de Gistaín, drenados por los ríos Ésera y Cinqueta respectivamente, que articulan el relieve, hendiendo profundos valles y resaltando el imponente volumen montañoso de este macizo. Forma un 
elevado cordal de dirección SO-NE, desde el collado de Sahún hasta el collado de Gistaín, que supera los $3.000 \mathrm{~m}$ de altitud en más de veinte picos. Todo el sector estudiado se enmarca en el grupo de Posets o Llardana, entre el Pico Posets y la Tuca de la Paúl (2.699 m), en los circos glaciares de Posets y la Paúl. La litología condiciona la morfología de los circos, individualizados, encajados y circulares, con la presencia de un estrato calizo vertical y continuo que configura la sucesión de circos escarpados desde de los Gemelos hasta Montidiego. En la Coma de la Paúl, limitada al norte por un umbral calcáreo, el arroyo proglaciar se sume en la cubeta glaciokárstica del mismo nombre, caracterizada por sus formas suaves derivadas de la abrasión glaciar y con extensos taludes de derrubios en sus laderas. Sin embargo, en la vertiente meridional, en el valle de los Ibone, el granito determina la excavación glaciar de cubetas bien definidas con pronunciados umbrales. Al resguardo de las cumbres se mantienen el glaciar de la Paúl y el helero de Posets, y al pie de las paredes reposan las morrenas bien conservadas de la PEH (SERRANO et al., 2001, 2002; LUGON et al, 2004). Los montículos hielo de Posets se localizan sobre el glaciar rocoso del Posets (SERRANO et al. 1999, 2010, 2011) y en el frente de la morrena lateral de la Paúl.

\section{METODOLOGÍA}

Se han estudiado en detalle 10 montículos de hielo en su contexto geomorfológico y ambiental (TABLA 1), y se han cartografiado los de su entorno. Para el análisis y seguimiento de la actividad de los montículos de hielo y su relación con las condiciones térmicas atmosféricas y de la formación superficial se han aplicado técnicas de inventariado, cartografiado y control térmico de los suelos:

- Se ha elaborado una cartografía geomorfológica de detalle a escala $1 / 10.000$ como herramienta esencial para el inventario, distribución y localización de procesos y formas, y su relación espacial y altitudinal.

- Las características y variabilidad interanual de las condiciones térmicas ambientales y en el suelo son claves esenciales en la dinámica periglaciar de alta montaña, pues señalan su función como aislante o aportación de agua por fusión, el comportamiento térmico del suelo y los ciclos de hielo/deshielo. Estos factores condicionan en buena medida la dinámica de los montículos de hielo. Se han realizado mediciones de la temperatura basal del manto nival (BTS), mediante termómetro sonda modelo HI 955501, y para la monitorización del régimen térmico del suelo se han instalado termómetros de registro continuo sobre los montículos de hielo y en su entorno, (Ibuttons modelos DS1922L y DS1921G). En Alba se han instalado cuatro 
termoregistradores continuos durante el año hidrológico 2010-2011, y en la Coma de la Paúl seis termoregistradores durante los años hidrológicos 2009-2010 y 2010-2011. El comportamiento térmico del suelo en los montículos de hielo permite conocer el estado del agua en el suelo (líquido, permafrost, hielo estacional), para conocer su génesis y evolución dinámica anual en relación con las condiciones ambientales.

Se han obtenido las temperaturas media anual atmosférica y media anual del suelo y el rango térmico del suelo en el período de estabilidad térmica invernal (TABLA 2). Los datos de los termómetros permiten además conocer la evolución y durabilidad del manto nival.

- Analisis de los datos de dos telenivómetros (La Renclusa, a 2.180 m.; y Eriste, a $2.350 \mathrm{~m}$, de la Red SAIH-CHE) para comparar los datos térmicos del suelo con los datos atmosféricos. Se ha aplicado el gradiente térmico altitudinal desarrollado por FEUILLET y SELLIER (2008) y FEUILLET (2010) en la alta montaña pirenaica para determinar los límites morfoclimáticos y los cambios térmicos en función de la altitud.

- Se han calculado los índices de helada y de deshielo (Freezing and thawing index) y los ciclos de hielo deshielo, que aportan información sobre la existencia y localización del permafrost o la eficacia de la helada (GUODONG et al., 2003; FENGQING y YANGWEI, 2009).

Tabla 1. Características morfológicas de los montículos de hielo estudiados

\begin{tabular}{lccrrrrc}
\hline Zonas & Altitud & Montículos & Anchura & Largo & Altura & Forma & Vegetación \\
\hline \multirow{5}{*}{ Maladeta } & \multirow{2}{*}{2.900} & M.H. 1 & 0,96 & 1,05 & 0,85 & Cuspidiforme & Ausente \\
& & M.H. 2 & 0,85 & 1 & 0,35 & Cuspidiforme & Ausente \\
& & M.H. 3 & 0,90 & 1 & 0,50 & Circular & Incipiente \\
& & M.H. 4 & 0,65 & 0,65 & 0,30 & Circular & Ausente \\
\hline \multirow{6}{*}{ Posets } & \multirow{2}{*}{$2.670-2.700$} & M.H. 1A & 2,9 & 2,7 & 0,64 & Oval & Ausente \\
& & M.H. 2A & 1,7 & 1,68 & 0,34 & Circular & Ausente \\
& & M.H. 3A & 2,8 & 2,64 & 0,40 & Circular & Incipiente \\
& \multirow{2}{*}{$2.770-2.800$} & M.H. 4A & 2,1 & 1,1 & 0,50 & Oval & Colonización \\
\hline \multirow{2}{*}{} & & M.H. 1B & 3 & 2,9 & 0,50 & Circular & Incipiente \\
& & M.H. 2B & 1,7 & 1,53 & 0,39 & Circular & Incipiente \\
\hline
\end{tabular}

(Todas las medidas en metros)

FUENTE: Los autores

\section{LAS CARACTERÍSTICAS TÉRMICAS Y MORFODINÁMICA}

\section{IV.1. Los montículos de hielo de la Maladeta-Circo de Alba}

Los montículos de hielo de la Maladeta se localizan en la zona interior de la morrena frontal de la PEH a $2.911 \mathrm{~m}$ en el circo de Alba. El contexto dinámico 
se caracteriza por el proceso de deglaciación que ha derivado en la presencia del helero de Alba, y la existencia de crioturbación, con génesis de suelos ordenados y lóbulos de gelifluxión (FIGURA 2). El helero ha sido progresivamente enterrado, y sobre el manto de clastos tienen lugar intensos procesos criogénicos.

Figura 2. Localización de los Montículos de Hielo del circo de Alba.

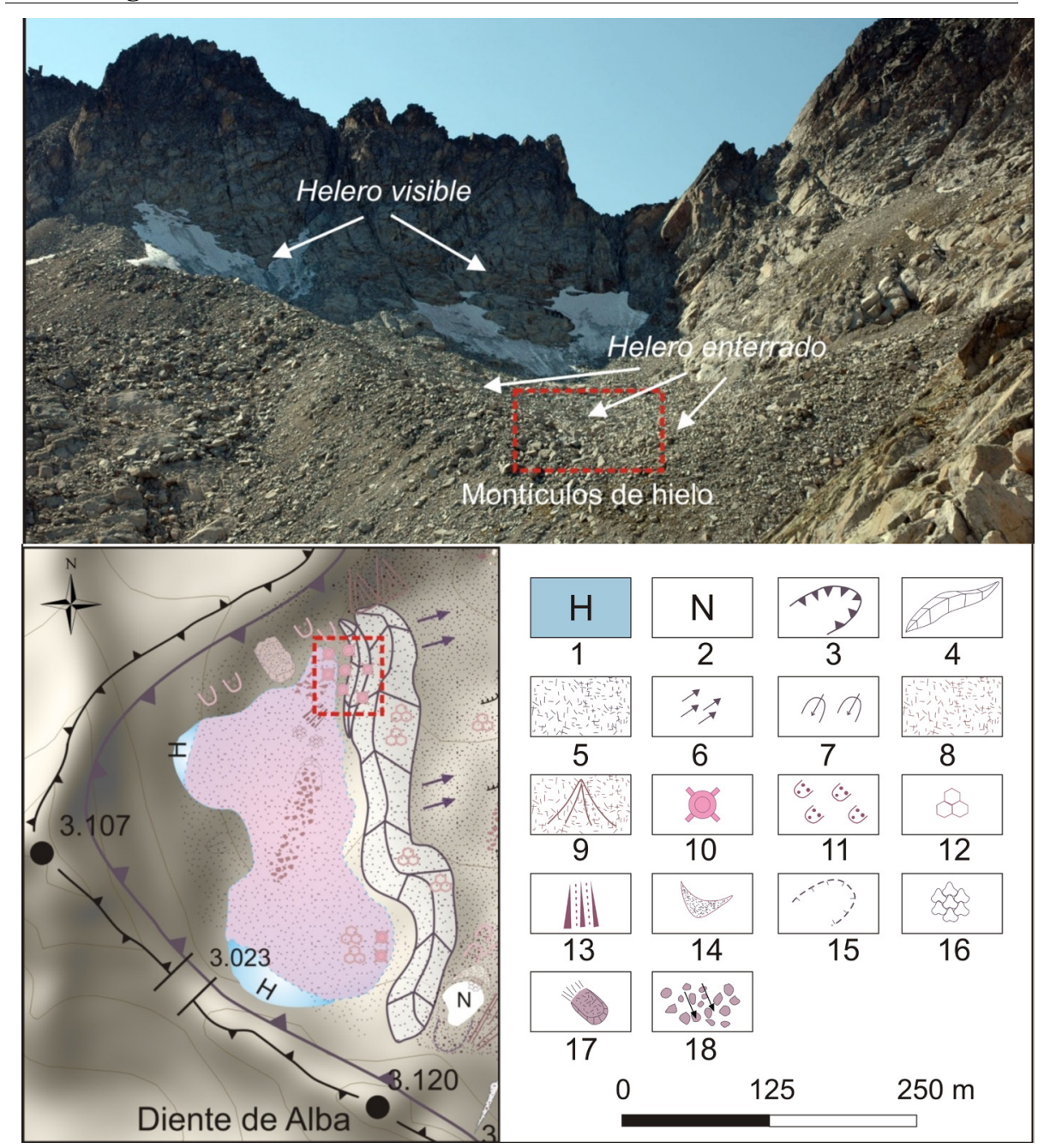

1, hielo. 2, nieve. 3, circo glaciar. 4, morrena. 5, till. 6, estrías glaciares. 7, rocas aborregadas. 8, taludes de derrubios. 9, conos de derrubios. 10, montículos de hielo. 11, lóbulos de gelifluxión. 12, suelos ordenados. 13, suelos estriados. 14, morrena de nevero. 15, nicho de nivación. 16, enlosado nival. 17, lóbulo protalud. 18, corriente de bloques FUENTE: Los autores. 
Los montículos de hielo se emplazan en zonas llanas con acumulación de materiales finos procedentes de la meteorización del granito y su posterior lavado, y se agrupan en familias de dos o tres ejemplares dispersas por la zona estudiada. Son formas cilíndricas, con la porción superior plana o cuspidiforme, y de pequeñas dimensiones, con una altura media entre 30 y $80 \mathrm{~cm}$ y una anchura $\sim 1$ m (TABLA 1).

Los montículos estan formados por un núcleo interior de hielo que en su proceso de segregación provoca la extrusión de materiales finos en la parte central - amontonamiento de clastos pequeños y heterométricos con matriz de finos-, y el derrubiamiento de los bloques de mayor tamaño hacia la periferia de la geoforma. Los derrubios que conforman los montículos de hielo presentan un incipiente ordenamiento, la mayor cantidad de material grueso reposa en la base de la forma y los más finos en la parte superior. Este leve ordenamiento es proporcional al período de durabilidad de los montículos de hielo, que es relativamente corto. La escasa o nula colonización vegetal, la presencia de hielo visible en superficie y la frescura de las geoformas permiten catalogarlas como plenamente activas. Las principales diferencias entre montículos estriban en su emplazamiento:

a) Localizados sobre el manto de derrubios que recubre el helero, en algún caso se produce una colada de finos que apenas deja huella de su deshinchamiento (FIGURA 3).

b) Localizados sobre y bajo la morrena de la PEH de Alba. Cuando el hielo se funde se produce un deshinchamiento total de la geoforma, fácilmente reconocibles por la acumulación de finos en la parte central y los bloques de gran tamaño tumbados con disposición planar y concéntrica sobre la morrena.

La temperatura media anual atmosférica obtenida a partir de los datos del telenivómetro de la Renclusa y la aplicación del gradiente altitudinal, es de $0,8^{\circ} \mathrm{C}$, y la temperatura media del suelo registrada por los termoregistradores, de $-0,3^{\circ} \mathrm{C}$. Durante 141 días, de finales de noviembre a marzo, el suelo permanece con temperatura media inferior a $-3^{\circ} \mathrm{C}$ (TABLA 2). Las mediciones BTS y la temperatura registrada en el período de estabilidad térmica ubican a esta zona en el rango térmico del suelo de $-4,5$ y $-2^{\circ} \mathrm{C}$, en ambiente con permafrost probable. El índice de helada es de $\mathrm{Ti}<0^{\circ} \mathrm{C} 183 \sum \%$ día y el índice de deshielo es de $\mathrm{Ti}>0^{\circ} \mathrm{C} 87 \sum$ /día, lo que confirma la existencia de permafrost probable (FIGURA 4). La cubierta nival se instala en octubre y se mantiene durante siete meses. 
Tabla 2. Características térmicas en el emplazamiento de los montículos de hielo

\begin{tabular}{|c|c|c|c|c|c|c|c|}
\hline Zonas & Altitud (m) & Montículos & $T$ & TMSSEP & TMAA & TMAS & E.T.I \\
\hline \multirow{4}{*}{ Maladeta } & \multirow{4}{*}{2.900} & M.H. 1 & 4,5 & \multirow{4}{*}{4} & \multirow{4}{*}{$-0,6$} & \multirow{4}{*}{$-0,3$} & \multirow{4}{*}{$(-2 / 4,5)$} \\
\hline & & M.H. 2 & 3,5 & & & & \\
\hline & & М.H. 3 & 3 & & & & \\
\hline & & M.H. 4 & 3,5 & & & & \\
\hline \multirow{5}{*}{ Posets } & \multirow{3}{*}{$2.670-2.700$} & M.H. 1A & 3,3 & & & & \multirow{5}{*}{$(0 /-1,5)$} \\
\hline & & M.H. 2A & 4,5 & 5,7 & 1,9 & 1,4 & \\
\hline & & $\begin{array}{l}\text { M.H. 3A } \\
\text { M.H. 4A }\end{array}$ & $\begin{array}{c}4,2 \\
4\end{array}$ & & & & \\
\hline & \multirow{2}{*}{$2.770-2.800$} & M.H. 1B & 5,4 & \multirow{2}{*}{5,3} & \multirow{2}{*}{1,3} & \multirow{2}{*}{1,7} & \\
\hline & & M.H. 2B & 6,1 & & & & \\
\hline
\end{tabular}

T, Temperatura del suelo a $15 \mathrm{~cm}$ de profundidad medido en el campo el 21 y 23 septiembre del año 2011, TMSSEP, Temperatura media en el suelo el 21 y 23 de septiembre; TMAA: Temperatura media anual atmosférica; TMAS: Temperatura media anual registrada por los termoregistradores continuos localizados en la geoforma o en zonas muy próximas; E.T.I: Rango térmico del suelo en el período de estabilidad térmica invernal.

FUENTE: Los autores

A finales de junio el balance térmico entre la atmósfera y la formación superficial es positivo, aumenta la temperatura del suelo y se produce la fusión total de la nieve, así como la circulación y congelación esporádica del agua en los derrubios superficiales, y procesos de crioturbación. Este fenómeno se produce en ambientes calcáreos de alta montaña como los Picos de Europa (GONZÁLEZ-TrueBA, 2007). Dependiendo de las variaciones interanuales pueden mantenerse o producirse su fusión y deshinchamiento, como ocurrió el año hidrológico 2008-2009 (FIGURA 3).

Figura 3. Detalle del montículo de hielo deshinchado y la colada de finos desarrollados sobre los derrubios que cubren el helero de Alba.

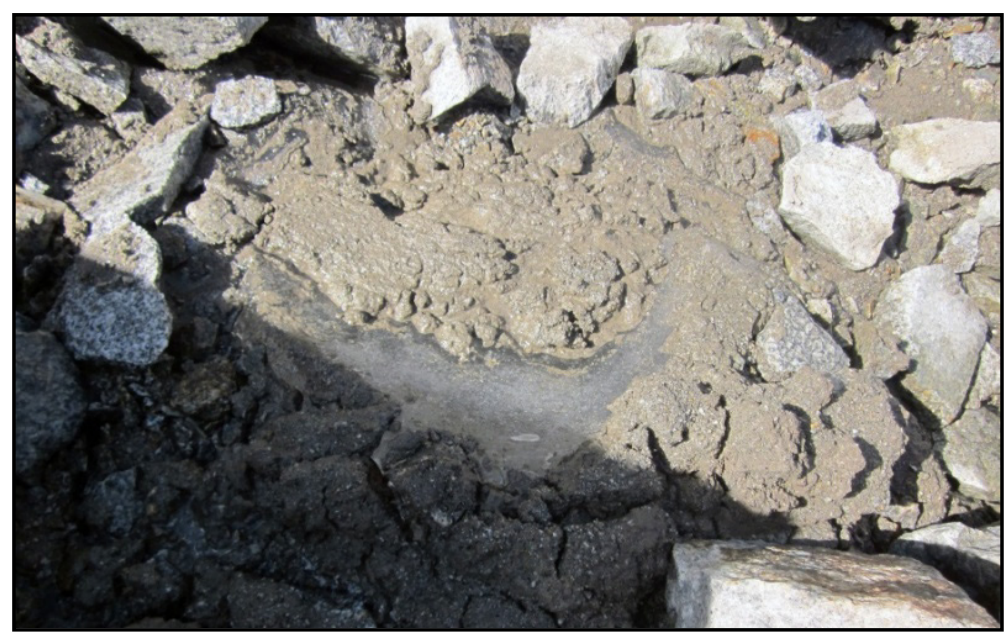

FUENTE: Fotografía de M. González García. 
Figura 4. Comportamiento térmico de la formación superficial en el Circo de Alba. El período de estabilidad del manto nival (4b) marca la existencia de permafrost.

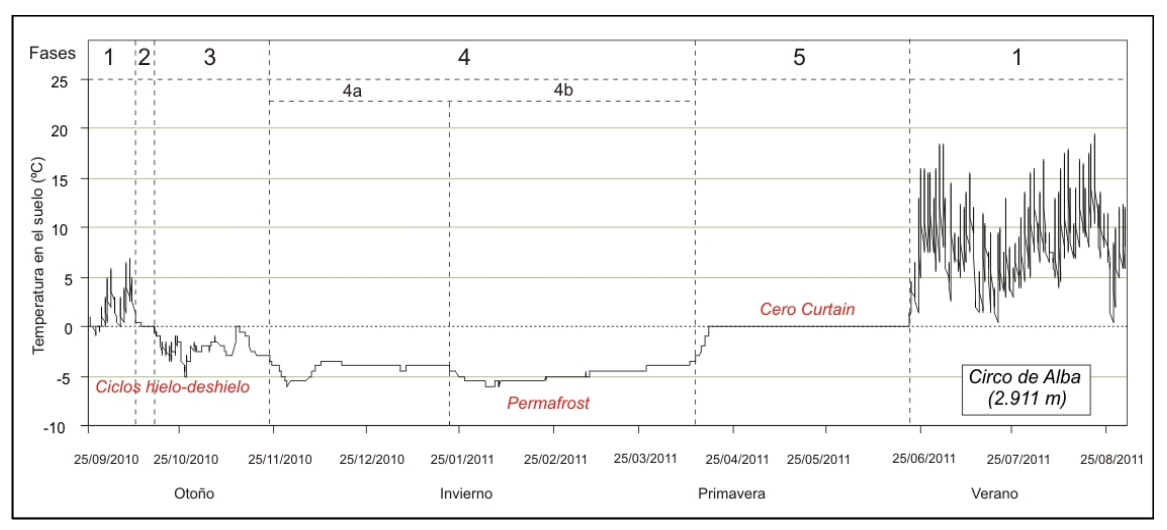

FUENTE: Los autores.

\section{IV.2. Los montículos de hielo del Circo de la Paúl y Posets}

En el macizo de Posets se han estudiado los montículos de hielo de la Coma de la Paúl y del circo de Posets. Los montículos de hielo de la Coma de la Paúl se localizan en dos zonas principales, Zona A y Zona B (FIGURA 5), ambas en sectores orientados al norte, protegidas de la radiación solar directa por la cresta de Bardamina durante buena parte del día, y con elevada disponibilidad hídrica procedente de fusión del manto nival en las laderas y del frente glaciar. La zona A se localiza entre 2.670 y $2.700 \mathrm{~m}$ sobre las morrenas de la PEH y en torno a ellas, en rellanos de sedimentos proglaciares remodelados por la nivación. La zona B se sitúa entre 2.770 y $2.800 \mathrm{~m}$, y los montículos de hielo se emplazan sobre los frentes morrénicos de la PEH. En ambos sectores se agrupan en familias y su tamaño es homogéneo, de 2 a $3 \mathrm{~m}$ de ancho y entre 34 y $64 \mathrm{~cm}$ de alto (TABLA 1), con formas cilíndricas u ovales y cúspide de tendencia planar. Su estructura se caracteriza por la presencia de un núcleo de clastos heterométricos congelados, parcialmente ordenados, con los clastos de menor tamaño arriba y los más grandes en la base, aunque también reposan sobre la porción superior bloques de mayor tamaño (FIGURA 6).

En el circo de Posets los montículos de hielo se sitúan sobre el glaciar rocoso de Posetselo asociados a un cuerpo helado, el del glaciar rocoso, con porciones llanas donde la circulación hídrica y la acumulación de humedad entre el recubrimiento de derrubios y el cuerpo helado propicia la génesis de hielo de segregación (SERRANO et al. 2010). Estos están estrechamente relacionados con la onda de frío emitida por el cuerpo helado infrayacente y la presencia de permafrost. 
Figura 5. Localización de los Montículos de Hielo del circo de la Paúl.
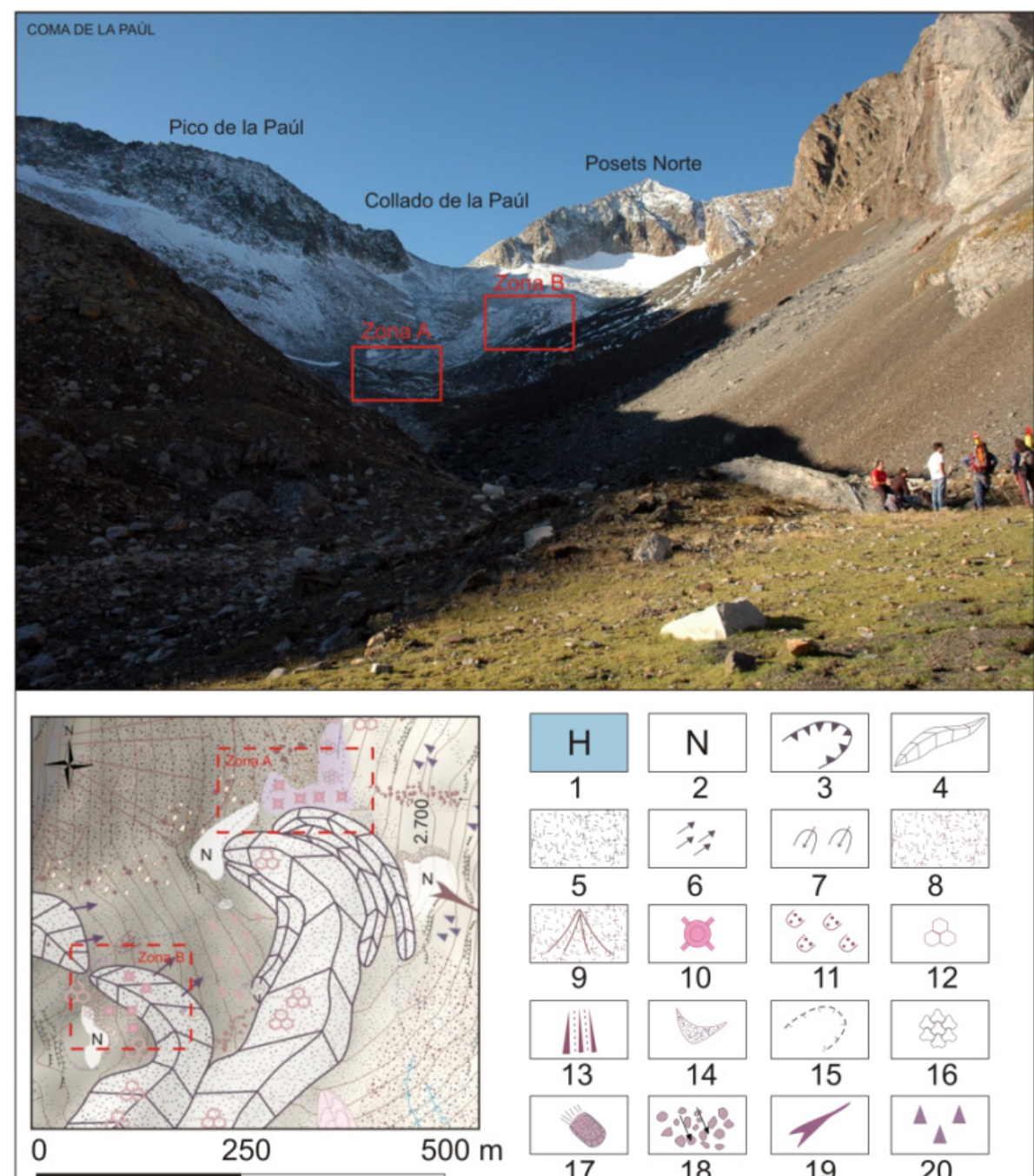

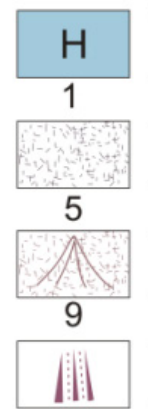

13

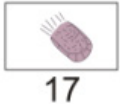

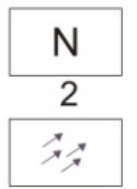

6

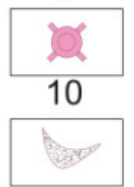

14

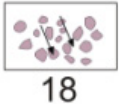

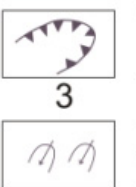

7

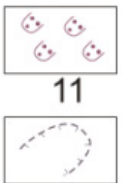

15

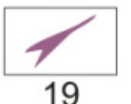

19

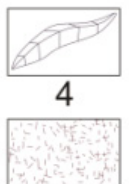

8

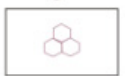

12

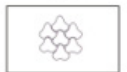

16

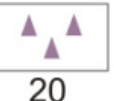

1, hielo. 2, nieve. 3, circo glaciar. 4, morrena. 5, till. 6, estrías glaciares. 7, rocas aborregadas. 8, taludes de derrubios. 9, conos de derrubios. 10, montículos de hielo. 11, lóbulos de gelifluxión. 12, suelos ordenados. 13, suelos estriados. 14, morrena de nevero. 15 , nicho de nivación. 16, enlosado nival. 17 , lóbulo protalud. 18, corriente de bloques. 19 , cono de aludes. 20, abrasión y pulido glaciar.

FUENTE: Los autores.

Las características geomorfológicas superficiales permiten establecer el grado de funcionalidad de los montículos de hielo: 
- Montículos de hielo inactivos: se caracterizan por el total deshinchamiento y la disposición planar de los clastos. La presencia de vegetación indica que son inactivas.

- Montículos de actividad atenuada: poseen una morfología irregular, la posición de los bloques en tendencia planar y una incipiente colonización vegetal (WASHBURN, 1973).

- Montículos de hielo activos: se caracterizan por su morfología regular, con clastos ordenados o en fase de reorganización por efecto del levantamiento por helada (hielo de segregación) y la gravedad. Los bloques adquieren una posición imbricada y reposan sobre el montículo (FIGURA 7).

Los sondeos de resistividad eléctrica han confirmado la existencia de hielo en la morrena lateral de la Paúl, que reduce su contenido de hielo hacia el frente (SERRANO et al. 2001; LUGON et al., 2004; DELALOYE, 2004), donde se concentra el mayor número de montículos de hielo activos. La temperatura media bianual de suelo en la Zona A (FIGURA 8) es de $1,4^{\circ} \mathrm{C}$ y en la Zona B de $1,7^{\circ} \mathrm{C}$ (TABLA 2), y durante el segundo año hidrológico se registraron un total de 2 ciclos de hielo-deshielo en el suelo en cada zona. Estos datos, junto a las medidas BTS confirman la inexistencia de permafrost a dichas altitudes. Sin embargo, los índices de helada y de deshielo pueden inducir a dudas sobre la existencia de permafrost. En la zona A $(2.680 \mathrm{~m})$ el índice de helada durante el período $2009-2010$ fue de $\mathrm{Ti}<0^{\circ} \mathrm{C} 70 \sum^{\circ}$ día y el índice de deshielo de $\mathrm{Ti}>0{ }^{\circ} \mathrm{C} 50 \sum \%$ día, en $2010-2011$ fue de $\mathrm{Ti}<0^{\circ} \mathrm{C} 267 \sum \%$ día y $\mathrm{Ti}>0^{\circ} \mathrm{C} 73$ $\Sigma^{\circ}$ día respectivamente; en la zona B $(2825 \mathrm{~m})$ durante el período 2009-2010 el índice de helada fue de $\mathrm{Ti}<0^{\circ} \mathrm{C} 10 \sum^{\circ}$ día y el índice de deshielo es de $\mathrm{Ti}>0^{\circ} \mathrm{C} 334 \sum^{\circ} /$ día, pero en el año hidrológico 2010-2011, se registró un índice de helada de $\mathrm{Ti}<0^{\circ} \mathrm{C} 251 \sum^{\circ}$ día y un índice de deshielo de $\mathrm{Ti}>0^{\circ} \mathrm{C} 86$ $\sum \%$ día. Atendiendo a la definición podríamos considerar que el índice del segundo período (2010-2011) refleja la existencia de permafrost, aunque con valores térmicos siempre más altos de $-2^{\circ} \mathrm{C}$.

El mantenimiento de la temperatura media anual del suelo entre 0 y $-1{ }^{\circ} \mathrm{C}$ durante el periodo protegido por la cubierta nival indica el posible congelamiento del agua retenida en el suelo. A su vez, hay considerables diferencias térmicas interanuales; si en la Zona A los días con temperaturas del suelo iguales o inferiores a cero (en su caso hasta $-2^{\circ} \mathrm{C}$ ) son superiores a los días en que la temperatura del suelo es superior a $0^{\circ} \mathrm{C}$, en la Zona $\mathrm{B}$, a mayor altitud, hay más días con temperaturas $>0^{\circ} \mathrm{C}$. Las condiciones ambientales de la Zona A, implican la mayor concentración de montículos de hielo plenamente funcionales. 
Figura 6. Características geomorfológicas superficiales y análisis granulométrico de un montículo de hielo activo.

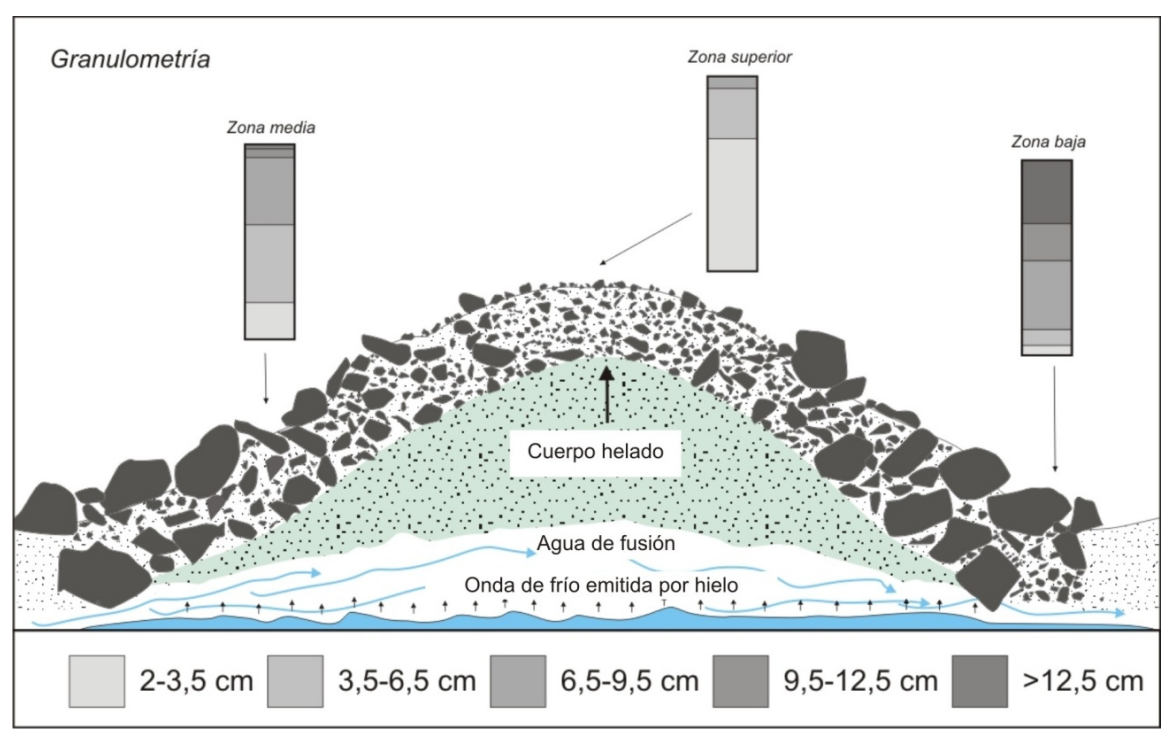

FUENTE: Los autores.

Figura 7. Montículo de hielo activo de la Zona A y montículo de hielo en fase de fusión y colapso de la Zona B.
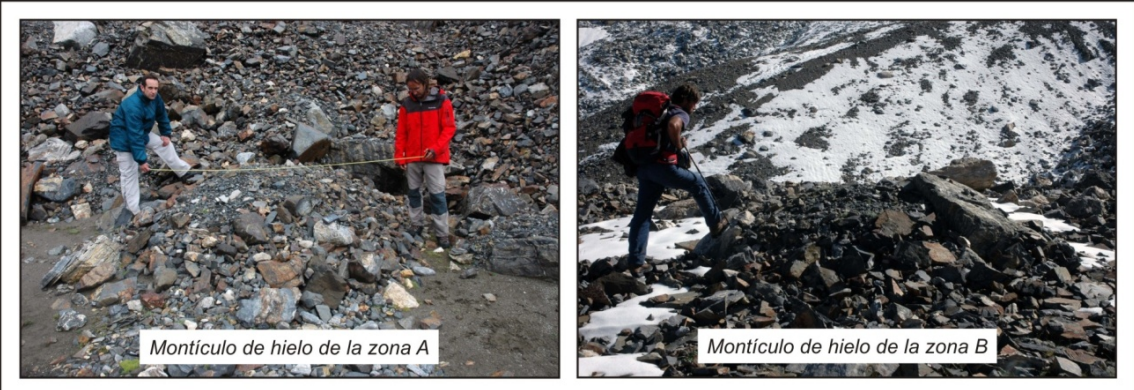

FUENTE: Los autores.

\section{IV.3. Morfodinámica de los montículos de hielo}

La presencia de hielo enterrado o un cuerpo helado es el factor esencial en la generación de los montículos de hielo en la Zona A de La Paúl, en Posets y en Alba, al tiempo que el manto nival (espesor y persistencia) y el efecto cero curtain favorecen su mantenimiento. El seguimiento térmico anual de los montículos de Alba y circo de Posets confirman la existencia de permafrost, 
en el primer caso asociado a un cuerpo de hielo enterrado, el helero de Alba, y en el segundo a una masa de hielo y cuerpo helado del glaciar rocoso de Posets. En los dos casos condicionan el régimen térmico de los derrubios suprayacentes (FIGURA 4). Son, pues, las rigurosas temperaturas del suelo, la permanencia del manto nival durante más de ocho meses y la acumulación de clastos con finos, los factores que propician la génesis de estas geoformas en las zonas más favorables para la retención de las aguas de fusión, siempre en sectores llanos.

El manto nival es un elemento también esencial en la morfogénesis de los montículos de hielo. La permanencia del manto nival hasta finales de junio (FIGURA 4) protege a la formación superficial de la radiación solar directa y favorece un proceso de retroalimentación que mantiene al suelo a una temperatura constante y asiste el mantenimiento de los montículos de hielo. Atendiendo a las gráficas de evolución térmica, en Alba el manto nival permanece durante más de ocho meses (oct-jun), en la Zona A de La Paúl 7 meses en el período 2009-2010 (nov-jun) y 8 meses en el 2010-2011 (oct-jun), y en la Zona B de La Paúl perdura durante 7 meses en ambos períodos (dic-jul y de nov-jun respectivamente). De este modo, entendemos que el suelo está aislado de las condiciones térmicas atmosféricas directas durante buena parte del año y el comportamiento térmico del suelo es independiente del atmosférico (FIGURA 8), de modo que su morfogénesis responde a la congelación de las aguas de fusión que circulan en la formación superficial sobre la masa de hielo o cuerpo helado.

En la morfodinámica de los montículos intervienen los factores topoclimáticos, la duración del manto nival, la presencia de hielo estacional, la textura de la formación superficial y la disponibilidad hídrica, favorecida por la existencia de rellanos mal drenados. El proceso de hinchamiento se produce por congelación del agua subsuperficial en lenta circulación por las zonas llanas, con génesis de hielo de segregación, y por la congelación de la humedad existente en los finos, que provocan el aumento del volumen a partir de la congelación del agua de ambos orígenes. El agua de fusión que se filtra en el suelo, a partir de marzo o abril, se congela por la onda de frío emitida por el helero o cuerpo helado, y mantiene el suelo a una temperatura de $0^{\circ} \mathrm{C}$ hasta finales del mes de junio. Cuando se funde el manto nival y hay una mayor exposición del hielo a la radiación solar (la fusión total de la cubierta nival es a principios de julio), la temperatura del suelo asciende y la tasa de fusión del núcleo de hielo aumenta, de modo que se produce un desequilibrio térmico y la desaparición del núcleo helado, con el progresivo colapso o deshinchamiento de la geoforma. 
Figura 8. Evolución térmica atmosférica y evolución térmica del suelo en la Zona A en ambos años hidrológico.
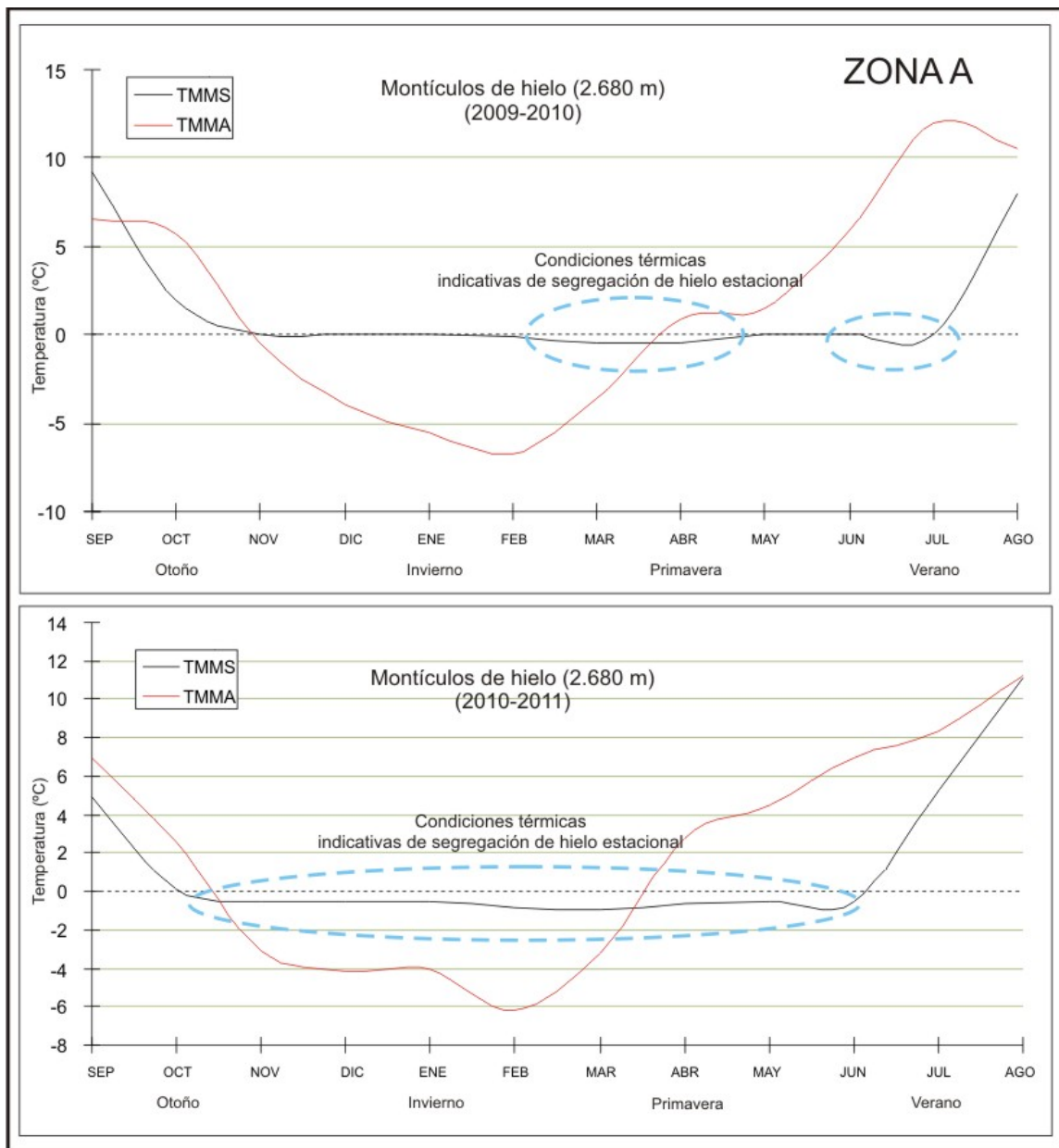

FUENTE: Los autores.

Los montículos tienen en su interior un cuerpo helado que extruye el material más fino y desplaza progresivamente los gruesos hacia las zonas externas y su base (FIGURA 9). De este modo se genera un ordenamiento de los clastos, con los gruesos al exterior y los finos al interior. Cuando el cuerpo de hielo queda expuesto a la radiación solar directa, aumenta el ritmo de fusión y la forma se desmantela. 
Figura 9. Esquema morfogenético de los montículos de hielo.

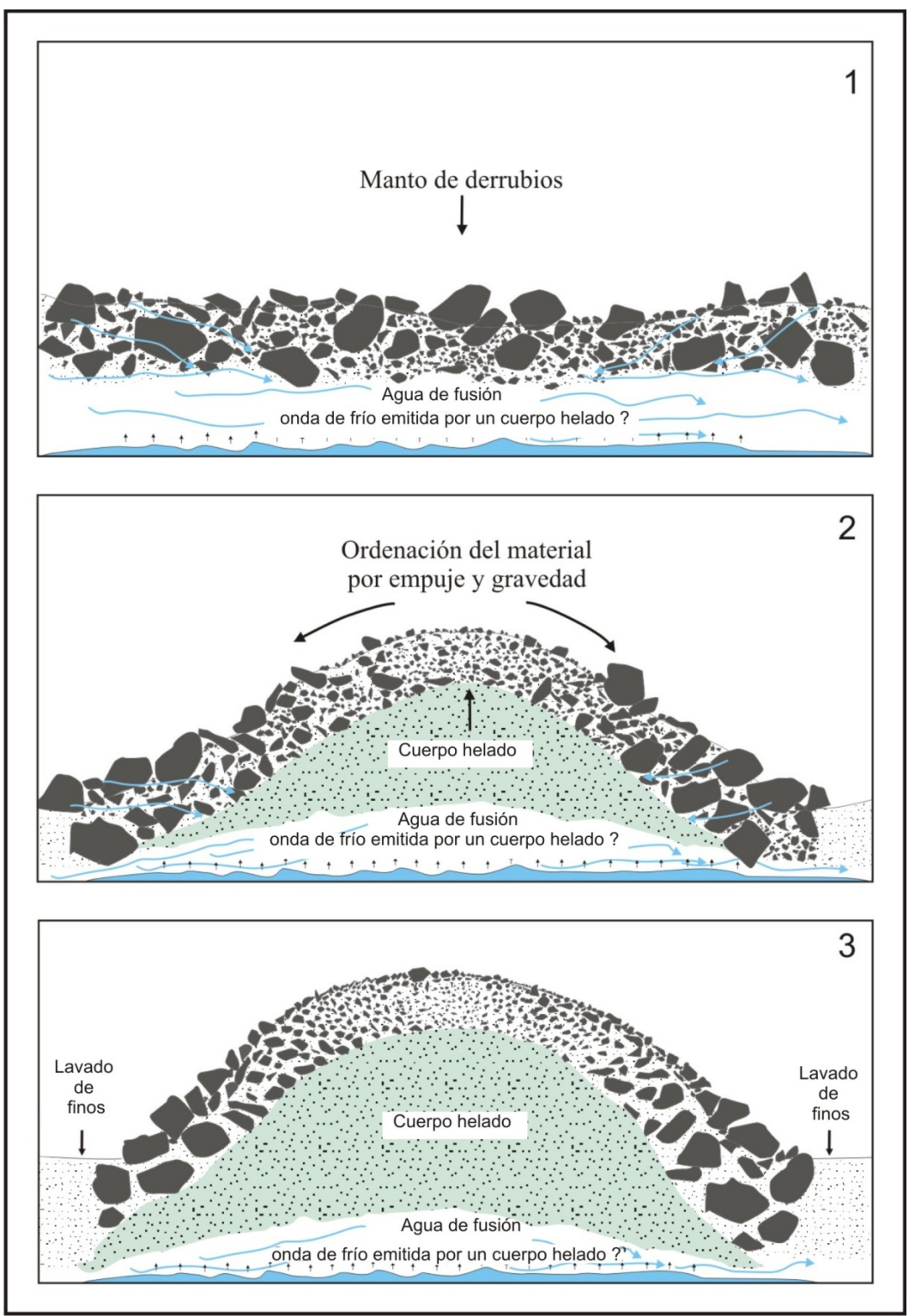

FUENTE: Los autores.

Por lo contrario, durante los años que las temperaturas son más bajas y el manto nival perdura hasta entrado el verano la geoforma perdura hasta el siguiente invierno, e incluso se generan nuevos montículos de hielo. En este 
sentido, las variaciones térmicas interanuales intervienen en la sucesión de fases de «construcción-destrucción» de las geoformas. La alternancia de derrubiamiento/ordenamiento favorecido por el hinchamiento, y deformación/desordenamiento favorecido por el deshinchamiento, además del reajuste ante la fusión del manto nival, retrabajan y removilizan los materiales. Se produce de este modo un ordenamiento, lo que ha conducido a su denominación en algunos casos como suelos ordenados incipientes, si bien los procesos son diferentes. En este caso la forma deriva de la acumulación de hielo de segregación, mientras en los suelos ordenados son los procesos de crioturbación asociados a las presiones diferenciales por congelación en la capa activa o en la porción superficial del suelo, los que generan la forma. Tampoco se ajusta a una interpretación como pingos en miniatura (ROUJANSKI, 2008), pues se producen sucesivos congelamientos y descongelamientos del depósito con génesis y destrucción de las formas, a menudo efímeras. En este caso los procesos de segregación de hielo si son similares, si bien de mucha menor entidad.

\section{CONCLUSIONES}

Los montículos de hielo se sitúan siempre por encima de los $2.600 \mathrm{~m}$, en el piso crionival. Generalmente se localizan en puntos con características topoclimáticas muy favorables: topografías llanas de la parte alta de los circos glaciares, próximas a las morrenas de la PEH y con presencia de finos.

En las zonas bajas, entre 2.600 y $2.850 \mathrm{~m}$, los factores topoclimáticos favorecen condiciones más rigurosas que las correspondientes a esa altitud (con rango térmico del suelo entre 0 y $-1,5^{\circ} \mathrm{C}$ ) y la mayor duración del manto nival (9 meses) mantiene el estado dinámico de los montículos de hielo, con temperaturas atmosféricas medias anuales entre $1 \mathrm{y}-1^{\circ} \mathrm{C}$, y temperaturas medias anuales del suelo entre 1,7 y $-0,3^{\circ} \mathrm{C}$. Su génesis está asociada al desarrollo de suelos helados estacionales o hielos residuales con recubrimiento de clastos.

A partir de 2.850-2.900 m, los rangos térmicos anuales del suelo se sitúan entre $-2 \mathrm{y}-4,5^{\circ} \mathrm{C}$, y su localización está asociada a la existencia de permafrost.

Por tanto, se puede establecer dos tipos de montículos de hielo por su génesis y en relación con la presencia de hielo en el subsuelo, estacional o permanente:

- Montículos de hielo en áreas con permafrost, donde la emisión de frío es directa desde el subsuelo. En este caso el ambiente con permafrost puede proceder de la presencia de un helero enterrado, capaz de emitir una onda de frío que mantiene temperaturas medias anuales del suelo en tono a $-0,3^{\circ} \mathrm{C}$, o de condiciones atmosféricas frías, con temperaturas medias invernales de hasta $-5^{\circ} \mathrm{C}$ (TMAA de $-0,6^{\circ} \mathrm{C}$ ), siempre por encima de los $2.900 \mathrm{~m}$. Los ligados a ambientes con permafrost y 
glaciares rocosos se localizan desde los $3.000 \mathrm{~m}$, con temperaturas medias anuales atmosféricas entre $-0,6$ y $-1,2^{\circ} \mathrm{C}$, y del suelo $<-1{ }^{\circ} \mathrm{C}$. Estos montículos se localizan en la franja altitudinal del permafrost probable.

Sobre los heleros, a las condiciones específicas derivadas de la altitud y de los factores topoclimáticos, se les une la onda de frío procedente de la masa de hielo relicta, subyacente al manto de derrubios, que congela las aguas de fusión nival y generan intensos procesos criogenéticos. Pero a su vez, dicho manto de derrubios ejerce una función protectora frente a la radiación solar directa en un proceso de retroalimentación que favorece un delicado equilibrio térmico y el manteniendo del helero. A ello se suma el carácter de aislante térmico ejercido por la nieve invernal y primaveral. Las temperaturas primaverales y la desprotección temprana del suelo de la cubierta nival, ocasiona la fusión del núcleo de hielo de los montículos, la generación de pequeñas coladas y el desmantelamiento o deshinchamiento de la geoforma.

- Montículos de hielo en áreas con hielo estacional, donde el frío procedente del aire y los factores ambientales y topoclimáticos (orientación norte, baja tasa de radiación solar, topografía abrupta, cubierta nival de más de 8 meses) favorecen el congelamiento del agua en el suelo. Los emplazamientos más favorables son las morrenas, o sus proximidades, donde se producen acumulaciones de finos. Las condiciones térmicas atmosféricas oscilan entre 2 y $1,3^{\circ} \mathrm{C}$, y las del suelo entre 1,5 y $1,7^{\circ} \mathrm{C}$. Estos montículos se localizan en la franja altitudinal del permafrost posible o inexistente, asociados a la presencia de suelos helados estacionales. La permanencia del manto nival favorece la actividad de los montículos de hielo, si bien debido a su baja cota y a la inexistencia de permafrost son más sensibles a los cambios ambientales estacionales.

Los montículos de hielo se localizan en el dominio crionival o alta montaña rocosa (piso periglaciar tipo), desde 2.600-2.700 m hasta 3.000$3.100 \mathrm{~m}$, entre los ambientes con permafrost posible y probable. En todo caso, constituyen indicadores geoambientales expresivos de un fenómeno periglaciar caracterizado por su variabilidad espacio-temporal, genética, según las fuentes de frío, y dinámica, con unas formas estables y otras sometidas a ciclos de construcción-destrucción. Su alta sensibilidad a los cambios ambientales implica una respuesta rápida y perfectamente detectable. Forman parte de un ambiente donde los procesos morfogenéticos están ligados a la criosfera, y su estudio permite extraer 
información sobre los procesos criogenéticos y su relación con los restos de hielo enterrado, remanentes de la PEH.

\section{AGRADECIMIENTOS}

Este trabajo ha sido financiado por el Ministerio de Economía y Competitividad, proyecto CGL-2015-68144-R. Agradecemos al Sistema Automático de Información Hidrológica (SAIH) de la Cuenca Hidrográfica del Ebro y a la Agencia Estatal de Meteorología (AEMET), ambas del Ministerio de Agricultura, Alimentación y Medio Ambiente, por los datos cedidos. Finalmente agradecemos la colaboración y ayuda de Fernando Berenguer, Manuel Gómez Lende, Vicente Gómez, Raúl Martín, Javier de Matías e Ibai Rico en las campañas de campo.

\section{BIBLIOGRAFÍA}

Delaloye, R. (2004): «Contribution à l'étude du pergélisol de montagne en zone marginale». GeoFocus, 10, $240 \mathrm{pp}$.

EVERDINGEN, R.O. Van (ed.) (2005). Multi-Language glossary of permafrost and related ground-ice terms. International Permafrost Association and National Snow and Ice Data Center/World Data Center for Glaciology, Boulder.

EVERDINGEN, R.O. Van (1978): «Frost mounds at Bear Rock, near Fort Norman, N.W.T., 1975-1976». Canadian Journal of Earth Sciences, 15, 263-276.

FENGQING, J. y YANWEI Z. (2011): «Freezing and thawing Index». En VIJAY P. Singh, PRATAP Singh y UMESh K. Haritashya (eds.). Encyclopedia of snow, ice and glaciers, Springer Verlag, Dordrecht, pp. 301.

GuOdonG, C., HAO, J., KeLI, W. y QINGBAI, W. (2003): «Thawing index and freezing index on the embankment surface in permafrost regions». Journal of Glaciology and Geocryology, 25(6), 603-607.

Feuillet, T. y SELLIER, D. (2008): «Observations sur la limiteinférieure de l'étagepériglaciairedans les Pyrénées centrales françaises (Bigorre)». Environnements périglaciaires, 15, 59-68.

FeuIlLet, T. (2010): Les formes périglaciaires dans les Pyrénées centrales françaises: analyse spatiale, chronologique et valorisation. $\mathrm{PhD}$ Thesis. Université de Nantes, 399 pp.

French, H. M. (2007): The Periglacial Environment. John Wiley \& Sons Ltd, Chichester.

García Ruíz, J.M., Bordonau, J., Martínez De Pisón, E. y Vilaplana, J. M. (1992): Mapa geomorfológico de Benasque (M. T. N. 180) (Huesca-Lérida), mapa 1:50.000. Geoformas Ediciones, Logroño.

GonZÁlez García, M., SERRANo CAÑADAS, E., SANJosé Blasco, J.J. y GonZÁlez TRUEBA, J.J., (2011): «Dinámica superficial y estado actual del glaciar rocoso de la Maladeta Occidental (Pirineos)». Cuadernos de Investigación Geográfica, 32, 81-94. 
GonZÁlez GARCíA, M. (2013): La alta montaña periglaciar en el Pirineo Central español: procesos, formas y condiciones ambientales. $\mathrm{PhD}$ Thesis, Facultad de Filosofía y Letras, Universidad de Málaga, Málaga.

GonzÁlez García, M., Serrano, E., SAnjosé, J.J. y GonzÁlez Trueba, J.J. (2016): «Surface dynamic of a protalus lobe in the temperate high mountain. Maladeta, Western Pyrenees». Catena, aceptado, en prensa.

GonzÁlez TruebA, J.J. (2007): El Paisaje Natural del Macizo Central de los Picos de Europa. Geomorfología y sus implicaciones geoecológicas en la alta montaña Cantábrica. CIMA-Gobierno de Cantabria, Santander, 333 pp.

González Trueba, J.J., Martín Moreno, R., Martínez De Pisón, E. y Serrano, E. (2008). «Little Ice Age glaciation and current glaciers in the Iberian Peninsula». The Holocene, 18, 551-568.

Lugon, R., Delaloye, R., Serrano, E., Reynard, E., Lambiel, C. y GonzÁlez TRUEBA, J.J. (2004): «Permafrost and Little Ice Age relationships, Posets massif, Central Pyrenees, Spain». Permafrost and Periglacial Processes, 15, 207-220.

MACKAY, J.R. (1973): "The growth of pingos, western Artic coast, Canada». Canadian Journal of Earth Sciences, 10 (6), 979-1004.

MACKAY, J.R. (1980): «Pingo ice of the western Arctic coast, Canada». Canadian Journal of Earth Sciences, 22 (10), 1452-1464.

MACKAY, J.R. (1998): «Pingo growth and collapse, Tuktoyaktuk Peninsula area, western Arctic coast, Canada: a long-term field study». Géographie Physique et Quaternaire, 52, 271-323.

MARTínEZ DE Pisón, E. (1989): «Morfología glaciar del valle de Benasque (Pirineo aragonés)». Ería, 18, 51-64.

MARTínEZ DE PISÓN, E. (1990): «Morfoestructuras del valle de Benasque (Pirineo Aragonés)». Anales de Geografía de la Universidad Complutense, 10, 121-148.

MARTíneZ De PISÓN, E. (1986): «El macizo granítico del Aneto». En B. Tello y E. Martínez de Pisón (Dir.), Atlas de Geomorfología, Alianza Editorial, Madrid, pp. $17-26$

POLLARD, W.H. (1988): «Seasonal frost mounds». En M.J. Clark (ed.). Advances in Periglacial Geomorphology, John Wiley and Sons, New York, pp.201-229.

POLLARD, W.H. (1991): «Seasonal frost mounds». The Canadian Geographer, 35 (2), 214-218.

RoUJANSKI, V. (2008). «Stone Frost Mounds in Shallow Bedrock Depressions at Lady Franklin Point, Victoria Island, Nunavut, Canada». En Ninth International Conference on Permafrost, University of Alaska-Fairbanks, IPA, pp. 1537-1542.

SERrAno, E. y AGUdo, C. (1998): «Glaciares rocosos activos de los Pirineos. Implicaciones ambientales». En Gómez Ortíz, A. (ed.). Procesos Biofísicos Actuales en Medios Fríos, Universitat de Barcelona, Barcelona, pp.133-154.

Serrano, E., Agudo, C. y Martínez De Pisón, E. (1999): «Rock glacier in the Pyrenees». Permafrost and Periglacial Processes, 10, 101-106.

SERrano, E., Martínez DE PISÓn, E. y Agudo, C. (2000): «El medio periglaciar de alta montaña en el Pirineo central: aportaciones recientes». En Procesos y formas periglaciares en la alta montaña mediterránea, Instituto de Estudios Turolenses, Teruel, 45-62. 
Serrano, E., Agudo, C., Delaloye, R. y González Trueba, J.J. (2001): «Permafrost distribution in the Posets massif, Central Pyrenees». Norwegian Journal of Geography, 55, 245-252.

Serrano, E., Agudo, C. y GonzÁlez TruebA, J.J. (2002): «La deglaciación de la alta montaña. Morfología, evolución y fases morfogenéticas glaciares en el macizo de Posets (Pirineo Aragonés)». Cuaternario y Geomorfología, 16 (1-4), 111-126.

SERRANO, E., SANJOSÉ, J.J. y AGUDO, C. (2006): «Rock glacier dynamic in a marginal periglacial high mountain environment: flow, movement (1991-2000) and structure of the Argualas rock glacier». Geomorphology, 74, 285-296.

Serrano, E., Morales, C., GonzÁlez Trueba, J.J. y Martin Moreno, R. (2009): «Cartografía del permafrost de montaña en los Pirineos españoles». Finisterra, 87, 45-54.

Serrano, E., SANJosÉ, J.J. y GonZÁlez TrueBA, J.J. (2010a): «Rock glaciers dynamics in marginal periglaciar environments». Earth Surface Processes and Landforms, 35 (11), 1302-1314.

Serrano, E., SAnjosé, J.J., Atkinson, A., Del Río, M., González García, M., GonzÁlez TRUEBA, J.J. y MARTín, R. (2010B): «Protalus lobe dynamic on Pyrenean high mountain». Third European Conference on Permafrost. Thermal State of Frozen Ground in a Changing Climate During the IPY. Svalbard, Noruega, IPA, abstract pp. 135.

Serrano, E., GonZÁlez TruebA, J.J. y SANJosé J.J. (2011): «Dinámica, evolución y estructura de los glaciares rocosos de los Pirineos». Cuadernos de Investigación Geográfica, 37 (2), 145-170.

WASHBURN, A.L. (1973): Periglacial processes and environments. Arnold, 320 pp. 